\title{
EFFECT OF HYDRATION AND PACKING ORDER ON LARGE UNILAMELLAR VESICLE FUSION: THE ROLE OF CHOLESTEROL
}

\author{
FRANCISCO J. CUEVAS ${ }^{1^{*}}$, CAROLINA A. VALLE $E^{2}$ AND LUIS F. AGUILAR

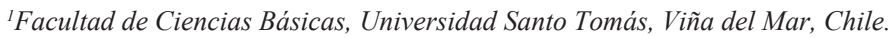 \\ ${ }^{2}$ Instituto de Química, Pontificia Universidad Católica de Valparaíso, Valparaíso, Chile
}

\begin{abstract}
Several studies examining vesicle fusion have been reported in last decades and have established a number of factors favoring the process of vesicle fusion. To determine whether variations to the physicochemical properties of the membrane affect the process of vesicle fusion, we worked with binary and ternary mixtures of large unilamellar vesicles (LUVs). The selected binary models were dioleoyl phosphocholine-cholesterol (DOPC-chol) and disteraroyl phosphocholine-cholesterol (DSPC-chol), and the tertiary mixtures were phosphatidylcholine-phsophatidylethanolamine-cholesterol (PC-PE-Chol); phosphatidylcholine-sphingomyelincholesterol (PC-SM-Chol); and phosphatidylcholine-phosphatidylserine-cholesterol (PC-PS-Chol). For all these models, the effect of cholesterol content on the lamella physicochemical properties was determined using 1,6-diphenyl-1,3,5-hexatriene (DPH) anisotropy, generalized polarization of 2-dimethylamino6-lauroylnaphthalene (Laurdan), and DPH fluorescence lifetime. To determine whether fusion of these vesicles varied according to lipid composition, the \% mixing content and the \% leakage were determined. Examining membrane incorporation using fluorescence steady-state and time-resolved probe assays in the models indicated that cholesterol content affected packing order and lamellar hydration. In most of the models, nonmonotonic variations were observed for these parameters, and these variations could be interpreted as increases in the proportion of ordered microdomains. When the proportion of these domains is higher, the packing order increases, and the lamellar water decrease. Similarly, the \% mixing, which was assessed as a fusion parameter, also exhibited nonmonotonic behavior, indicating that the fusion process is enhanced at these concentrations of cholesterol. However, DSPC vesicles do not merge, so more than the presence of microdomains is required to stabilize fusion.
\end{abstract}

Abbreviations: DPH, 1,6-diphenyl-1,3,5-hexatriene; GP, generalized polarization; Laurdan, 2-dimethylamino-6-lauroylnaphthalene;LUV, large unilamellar vesicles; DOPC, 1,2-dioleoyl-sn-glycero-e-phosphocholine; DSPC, 1,2-disteraroyl-sn-glycero-3-phosphocholine;PC, phosphatidylcholine; PE, phsophatidylethanolamine; SM, sphingomyelin; PS, phosphatidylserine.

\section{INTRODUCTION}

Vesicle fusion is required for several biological processes, such as vesicular trafficking, synaptic transmission, cell-cell fusion and viral fusion ${ }^{1}$. Although this process is regulated by proteins in vivo, the basic mechanism is determined by lipid-lipid interactions ${ }^{2}$. In addition to cellular functions, vesicle fusion is also important for other types of processes, such as using vesicles as carriers $\stackrel{3-8}{-}$. In vivo, SNARE proteins play a central role in vesicle fusion, and these proteins appear to be associated with rafts to regulate the merging process $\frac{9-11}{2}$. Vesicle-vesicle fusion studies have indicated that other agents, such as peptides $\underline{12}, \underline{13}$ or poly(ethylene glycol) (PEG) $\stackrel{14}{4}$, are required in the absence of these intrinsic proteins. Several mechanisms explaining how PEG accomplishes fusion have been suggested. Lentz (2007) $\frac{15}{5}$ summarized these mechanisms as follows: increasing surface tension, altering structure and dielectric properties of bulk water, altering the bilayer molecular order at the point of contact, promoting volume exclusion-induced aggregation and dehydration, inducing a non-bilayer structure, inducing bilayer-destabilizing phase separation, and producing compression followed by expansive osmotic stress on the membrane vesicles upon dilution, leading the contained impurities to disrupt the membrane. To understand the fusion process, the "stalk model" has been developed over the years to explain the merging phenomenon $16-$ 18. In this model, at least two intermediates have been reported. This model describes the formation of the first intermediate for the stalk followed by the formation of a second intermediate (trans-monolayer contact, TMC) that subsequently forms the pore and produces mixing 15 .All PEG-mediated fusion studies recently published have concluded that the physicochemical properties of the lipid composition mediates the formation of any of the intermediates $\frac{15}{5}$, 19. For instance, Talbot et al. (1997) ${ }^{20}$ explained that the combined effects of high membrane curvature and extensive chain unsaturation enhanced rate of lipid motion in the upper region of the bilayer, reflecting decreased packing density in the outer leaflet of unsaturated bilayers and an increased probability of fusion and complementing. Haque et al. $\frac{19}{\text { explained that each lipid seems }}$ to have a specific role in membrane fusion. For example, phosphatidylcholine (PC) has a hydrated headgroup that inhibits fusion in model membranes, PE is cone-shaped and promotes the bilayer-to-hexagonal phase transition that might facilitate membrane fusion, and SM has shape and hydration characteristics similar to PC.

Cholesterol is a lipid that is important in many membrane processes and plays a decisive role in the physicochemical properties of the membrane. Recently, a series of studies have reported that cholesterol regulates the fusion process between vesicles and cells, and most of these papers report that these system mergers are associated with the formation of cholesterol-rich domains or "rafts" that favor the fusion process $21-24$. In a vesicle-vesicle PEG-dependent fusion process, cholesterol has been reported to induce negative curvature that generates instability in some systems and favors breaking over merger in some

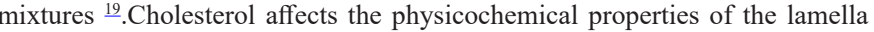
by dramatically increasing the order of acyl chains and inducing regular distribution domains. Several conceptual theories explain these phenomena as the superlattice model or the umbrella model $\stackrel{25}{2}$. In the superlattice theory, cholesterol molecules tend to be regularly distributed in a hexagonal host lipid lattice; however, irregular distributions always coexist with regular distributions

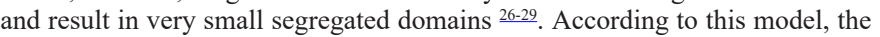
ratio of regular to irregular regions reaches local maxima at critical cholesterol molar fractions, and we suggest that in these states, the domain boundary surfaces also reach local maxima $\underline{30}, \underline{31}$. Furthermore, it is hypothesized that the process of vesicle-vesicle fusion is affected by the physicochemical properties of the lamella, which are influenced by changes in cholesterol content. Accordingly, in this work, large unilamellar vesicles (LUVs) made of binary mixtures of DSPC and DOPC with different cholesterol content or ternary mixtures of $\mathrm{PC} / \mathrm{PE}$ were examined. $\mathrm{PC} / \mathrm{SM}$ and $\mathrm{PC} / \mathrm{PS}$ mixtures with different proportions of the same molecule were also used. The concentrations were chosen in line with the prediction of microdomain formation by the superlattice theory to determine whether changes in the properties of the lamella affect the PEG-mediated melting process.

\section{MATERIALS AND METHODS}

Materials

1,6-Diphenyl-1,3,5-hexatriene (DPH) and 2-(dimethylamino)-6lauroylnaphthalene (Laurdan) were obtained from Molecular Probes (Eugene, OR, USA). All lipids and cholesterol were obtained from Avanti Polar Lipids (Alabaster, AL, USA). All other chemicals were of analytical or spectroscopic grade and were obtained from commercial sources.

Large Unilamellar Vesicle (LUV) Preparation

LUVs were prepared using the high-pressure extrusion method. Typically, different solid lipids were solubilized in chloroform to obtain stock solutions of each, and the volumes needed for different relative concentrations were extracted to prepare the respective vesicles. These mixtures were dried under a nitrogen stream to obtain a homogeneous film and suspended in the appropriate assay buffer $\left(\mathrm{N}_{2}\right.$ saturated) above the lipid order/disorder phase transition, to 
a final concentration of $0.35 \mathrm{mM}$. For Tb/DPA, the following measurements described by Talbot et al. .20 were used: the lipid was suspended in either 150 $\mathrm{mM}$ DPA or $15 \mathrm{mM} \mathrm{TbCl}_{3}$ plus $150 \mathrm{mM}$ sodium citrate and $2 \mathrm{mM} \mathrm{TES} / 2 \mathrm{mM}$ histidine, $\mathrm{pH}$ 7.4.For the leakage experiments, vesicles prepared in $7.5 \mathrm{mM}$ $\mathrm{TbCl} 3,75 \mathrm{mM}$ sodium citrate, $75 \mathrm{mM}$ DPA, $2 \mathrm{mM}$ TES, and $2 \mathrm{mM}$ histidine at $\mathrm{pH} 7.4$ were used. In all cases, the multilamellar vesicles were subjected to 15 freeze-thaw cycles in liquid nitrogen and then extruded ten times through a 100 -nm polycarbonate filter.

\section{Fluorescence Spectroscopic Measurements}

Steady-state and time-resolved fluorescence measurements were performed on a K-2 multifrequency phase and modulation spectrofluorometer (ISS, Champaign, IL, USA). The instrument was equipped with a GlanThompson polarizer. For both probes, the excitation light was provided by modulable ISS 375-nm LED laser. The emission was measured through Schot KV-399 and WG-420 long band-pass filters. The lifetime measurements were performed with the polarizer oriented in the "magic angle" condition ${ }^{32}$. To determine anisotropic decay, differential phase angles and modulation ratios were obtained using parallel and perpendicularly oriented sinusoidal polarized emission. The phase and modulation values were obtained as previously described 33, 34. Dimethyl-POPOP (1,4-bis[2]4-methyl-5-phenyloxazoly benzene) in ethanol ( $\tau=1.45 \mathrm{~ns}$ ) was used as a reference for intensity decay. The generalized polarization of Laurdan was utilized to assess fluorescence spectral shifts, which were measured as previously described ${ }^{35}$. The data are presented as the mean values and the standard errors of the measurements of three independent samples. In all cases, Laurdan and DPH final concentrations was $1 \mu \mathrm{M}$.

Data Analysis

Time-resolved fluorescence data were analyzed using the $\mathrm{VINCI}^{\mathrm{TM}}$ software package (ISS, Champaign, IL, USA). The fitting function for lifetime measurements was the sum of a continuously distributed Lorentzian component and a discrete component that was fixed at $0.01 \mathrm{~ns}$ to account for scattered light $\frac{34}{}$.

\section{Content Mixing and Leakage Assay}

The Tb-DPA leakage and content mixing assay were based on assays described by Talbot et al. (1997) ${ }^{20}$. Different PEG percentages from $10 \%$ to $25 \%$ were utilized (data not show). The best fusion results were obtained from $20 \%$ PEG. Similar results were obtained with an ANTS-DPX system (data not show). The parameters were collected as described below.

\section{$\%$ Leakage}

According to what is reported by Talbot et al. (1997) $\stackrel{20}{ }$, when leakage of contents occurred, there was a drop in fluorescence due to the quenching by water of $\mathrm{Tb}^{3+}$ released from complexation with DPA due to dilution of the trapped compartment into external compartment. To quantify this process, the fluorescence intensity of the vesicle mixture coencapsulated with Tb-DPA
(Ifco $0 \%)$ and the intensities of these vesicles after the addition of sodium cholate detergent (Ifco $0 \%$ det) were measured. The same process was also applied in the presence of $20 \%$ PEG (Ifco $20 \%$, Ifco $20 \%$ det). Thus the $\%$ leakage is defined as:

$$
\% \text { Leakage }=\frac{(\text { Ifco } 0 \%-\text { Ifco } 0 \% \text {,det })-(\text { Ifco } 20 \%-(\text { Ifco } 20 \% \text {,det })}{(\text { Ifco } 0 \%-\text { Ifco } \% \text {,det })} \times 100
$$

$\%$ Mix (\% Content Mixing): This assays were carried out by incubation equal volumes of separate vesicle populations (each at a final lipid concentration of $0.35 \mathrm{mM}$ ) containing $\mathrm{Tb}^{3+}$ and DPA, respectively with buffer containing $\mathrm{x} \%$ PEG. After 5 min of incubation, the fluorescence intensity was read (Ifmez), and detergent sodium cholate detergent in the presence of $20 \%$ PEG was added to release the contents of the vesicles, and fluorescence intensity was again recorded (Ifmezdet). The mixing ratio was calculated as follows:

$$
\% \text { ContentMixing }=\frac{(\text { Ifmez }- \text { Ifmezdet })}{1 / 2(\text { Ifco } 0 \%-(\text { Ifco } 20 \%, \text { det })}
$$

All measurements, including both fluorescence and fusion measurements, were collected at $37^{\circ} \mathrm{C}$

\section{RESULTS}

To study the effect of lamellar physicochemical properties on the vesicle fusion process, LUVs of different lipid compositions were used. The LUV were made of the following mixtures: DOPC-Chol, DSPC-Chol, PC-PE-Chol, PC-SM-Chol, and PC-PS-Chol. All measurements were performed at $37^{\circ} \mathrm{C}$.

To study the effect of lipid composition, the physicochemical propertie were measured according to three fluorescent parameters: DPH steady state anisotropy, the generalized polarization of Laurdan, and the fluorescence lifetime of the DPH probe. The DPH probe was chosen over its derivatives because it allowed us to collect information describing the average of all lamellar hydrophobic regions. The results are described below.

\section{Steady-State Fluorescence Measurements}

The effect of lipid content on the membrane models were studied using DPH steady-state fluorescence anisotropy and Laurdan emission spectral shifts that were assessed according to the generalized polarization parameter, GP (Fig.1 and 2). DPH and Laurdan fluorescent dyes localize to different positions in the lipid bilayer, the acyl chain deep hydrophobic core and the hydrophobichydrophilic interphase, respectively, and therefore can report the effect of lipid composition at these two locations.

In general, changes to DPH anisotropy with increasing cholesterol content in the bilayer followed a general trend that has already been described in the literature $\underline{36}, \underline{37}$. The behavior of this parameter in the studied models is summarized below.

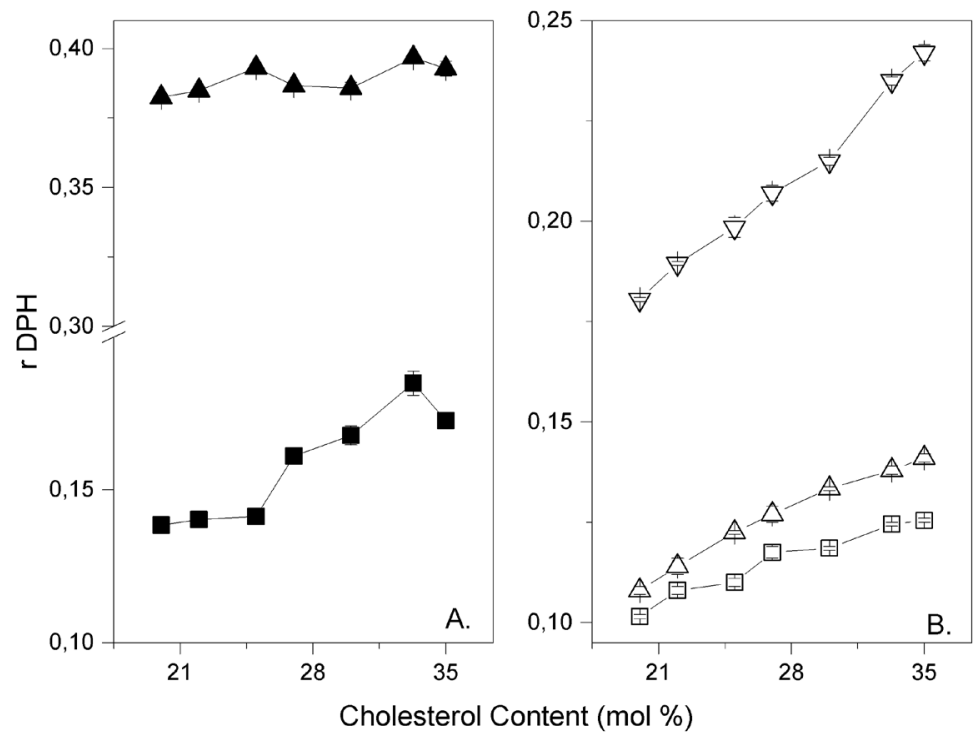

Figure 1: Effect of cholesterol on DPH anisotropy incorporated into large unilamellar vesicles containing A.-m- DOPC, $\Delta$ - DSPC and B. - $\square$ - PC:PE, $-\Delta-$ PC:SM, and - $\Delta$-PC:PS. Each point represents the average and standard error of triplicate data. 


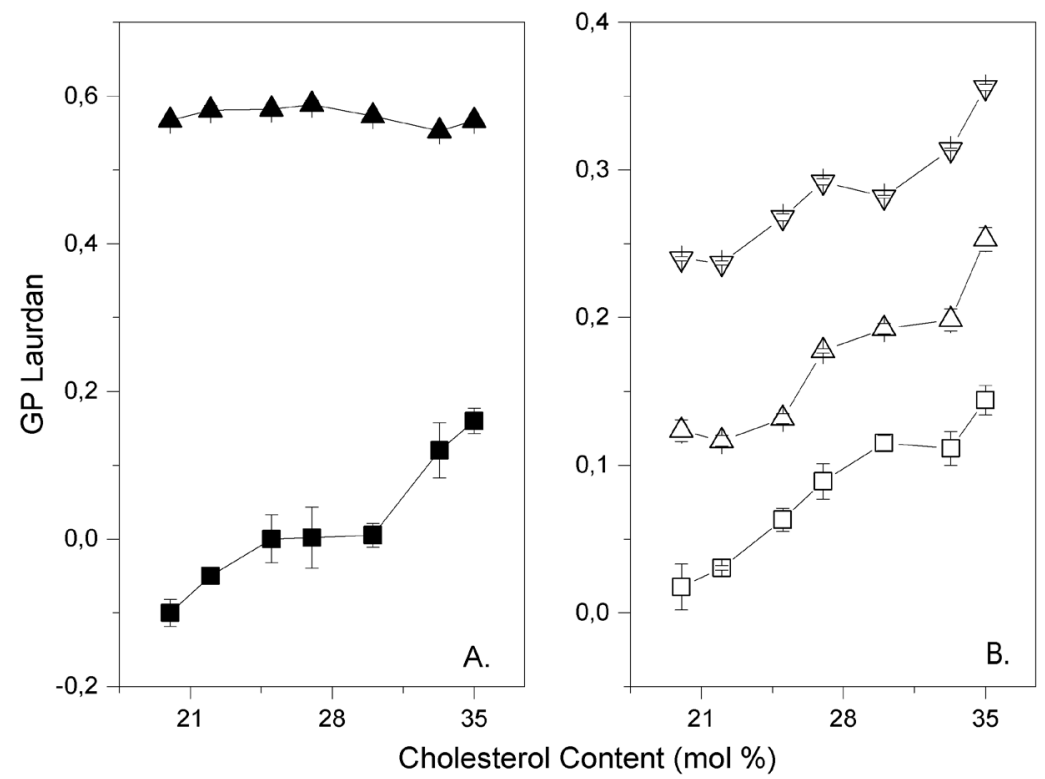

Figure 2: Effect of cholesterol on Laurdan GP incorporated into large unilamellar vesicles containing A.-m- DOPC, - $\mathbf{\Delta}$ DSPC and B. - $\square$ - PC:PE, $-\Delta-\mathrm{PC}: S M$, and $-\Delta$-PC:PS. Each point represents the average and standard error of triplicate data.

Comparing the anisotropies of binary mixtures formed from lipids with acyl chains of equal length in the presence or absence of unsaturation revealed that DSPC resulted in higher anisotropy values at any concentration of cholesterol (Fig. 1A). This finding indicated that DSPC-containing vesicles exhibited a higher packing order characteristic of the $l o$ phase. Increases in the cholesterol content resulted in small increases in anisotropy values in the DOPC-Chol model, which exhibited a small maximum near $33.3 \mathrm{~mol} \%$ of cholesterol. These peaks indicated that this concentration generated more local order in the lamella. The DSPC-Chol model did not show an increase similar to the DOPC-Chol model, probably because this system has a high packing order; however, a slight tendency near of $33.3 \mathrm{~mol} \%$ was similar to the DOPC-Chol system was observed. The presence of these local maxima could be due to the formation of submicroscopic domains.

Regarding the ternary mixtures, the effect of cholesterol over DPH anisotropy was higher than that observed in the binary mixtures. Moreover, this effect was more significant in the PC-PS-Chol mixtures, which exhibited anisotropy increases of approximately $40 \%$ (Fig. 1B). The increases in anisotropy in these mixtures were monotonic and did not exhibit the local maxima observed with the binary mixtures. Low values of the DPH anisotropy can be explained because the ternary mixtures are formed by natural lipids. The higher values in mixtures composed of PC:PS compared to the other two systems, indicate that the presence of charged lipid tends to generate a higher packing order.

Laurdan is used as a highly sensitive probe for the glycerol backbone of phospholipids. The spectral shift of the Laurdan probe (quantified with the GP parameter) correlate with the hydration and/or molecular dynamics of water molecules in the hydrophilic-hydrophobic interphase of the lamella ${ }^{35}$. For the binary mixtures (Fig. 2A), the GP values were higher for the DSPC mixture at all cholesterol concentrations, which indicated slower hydration or water molecular dynamics into the hydrophilic-hydrophobic interphase in these mixtures. Increases in the cholesterol content of these mixtures did not result in significant variation in this parameter, although with the DOPC-Chol mixture, the GP increased, indicating that cholesterol decreased hydration or the molecular dynamics in this region of the bilayer. These results are concordant with the peak order observed by DPH anisotropy.

The cholesterol effect was similar in the ternary mixtures. Increases in cholesterol content increased the GP value, indicating that the presence of this molecule in the membrane decreased the molecular dynamics or hydration of the hydrophobic-hydrophilic interphase (Fig. 2B). This pattern correlates with the above-mentioned results from Parasassi et al. .35. However, in these mixtures, the behavior was nonmonotonic, with a slope change near $33.3 \mathrm{~mol} \%$, indicating that cholesterol generates different molecular dynamic variations in water near this concentration.

Time-Resolved Fluorescence Measurements

The fluorescence lifetime of DPH embedded in the vesicle bilayer was also examined in the same systems (Tables 1 and 2). All DPH lifetime data were analyzed using a Lorentzian distribution (Methods), and the center of the distribution $\left(\tau_{c}\right)$ was reported as the fluorophore lifetime.

Table 1. Effect of cholesterol on DPH fluorescence lifetime distribution centers (ns) in large unilamellar vesicles from binary models containing DOPC-Chol and DSPC-Chol.

\begin{tabular}{|c|c|c|}
\hline $\begin{array}{c}\text { Cholesterol } \\
\text { content }\end{array}$ & DOPC & DSPC \\
\hline 20 & $7.56 \pm 0.02$ & $8.27 \pm 0.03$ \\
\hline 22 & $7.72 \pm 0.01$ & $8.33 \pm 0.03$ \\
\hline 25 & $7.96 \pm 0.03$ & $8.61 \pm 0.08$ \\
\hline 27 & $8.22 \pm 0.08$ & $8.60 \pm 0.08$ \\
\hline 32 & $8.42 \pm 0.02$ & $8.65 \pm 0.06$ \\
\hline $\mathbf{3 3 . 3}$ & $\mathbf{8 . 7 2} \pm \mathbf{0 . 0 4}$ & $\mathbf{8 . 9 2} \pm \mathbf{0 . 0 5}$ \\
\hline 35 & $8.54 \pm 0.02$ & $8.82 \pm 0.04$ \\
\hline
\end{tabular}

Each point represents the average of triplicate measurements of the Lorentzian distribution center, which had widths that ranged between 1.6 and $2.5 \mathrm{~ns}$. All measurements had c2values less than 1.5. The observed local maximum at $33.3 \mathrm{~mol} \%$ is bold (see text). 
Table 2. Effects of cholesterol on DPH fluorescence lifetime distribution centers (ns) in large unilamellar vesicles from the ternary models containing PC-PE-Chol, PC-SM-Chol, and PC-PS-Chol.

\begin{tabular}{|c|c|c|c|}
\hline $\begin{array}{c}\text { Cholesterol } \\
\text { content }\end{array}$ & PC:PE & PC:SM & PC:PS \\
\hline 20 & $6.75 \pm 0.07$ & $7.65 \pm 0.07$ & $8.80 \pm 0.03$ \\
\hline 22 & $6.51 \pm 0.09$ & $8.17 \pm 0.05$ & $9.01 \pm 0.09$ \\
\hline 25 & $6.91 \pm 0.05$ & $8.32 \pm 0.06$ & $9.34 \pm 0.05$ \\
\hline 27 & $6.91 \pm 0.04$ & $8.25 \pm 0.03$ & $8.89 \pm 0.04$ \\
\hline 32 & $7.47 \pm 0.05$ & $8.07 \pm 0.07$ & $9.24 \pm 0.05$ \\
\hline $\mathbf{3 3 . 3}$ & $\mathbf{7 . 5 5} \pm \mathbf{0 . 0 3}$ & $\mathbf{8 . 5 1} \pm \mathbf{0 . 0 5}$ & $\mathbf{9 . 6 3} \pm \mathbf{0 . 0 6}$ \\
\hline 35 & $7.25 \pm 0.17$ & $8.26 \pm 0.05$ & $8.96 \pm 0.07$ \\
\hline
\end{tabular}

Each point represents the average of triplicate measurements from the Lorentzian distribution center, which had widths that ranged between 1.8 and $2.9 \mathrm{~ns}$. All measurements had c2values less than 1.5. The observed local maximum at $33.3 \mathrm{~mol} \%$ is bold (see text).

The lifetime of DPH probes incorporated into membrane models before variation was measured as cholesterol was increased. For the binary mixtures, increases in cholesterol content caused significant increases in the DPH lifetimes in both models (Table 1). In general, the mixture containing DSPCChol exhibited greater lifetimes at all cholesterol concentrations. These findings correlate with steady-state results and indicate that for this mixture, there was reduced water molecule penetration into the lamellar depths due to the absence of unsaturations, and this model was below its phase transition temperature. In addition, the increase in lifetime as a function of cholesterol content was greater for the DOPC-Chol mixture than for the DSPC-Chol mixture (15\% and $8 \%$, respectively), likely because the mixture containing DSPC had less water in the center of the lamella. In contrast, both models showed nonmonotonic behavior and presented a significant maximum near $33.3 \mathrm{~mol} \%$, indicating a local maximum near the cholesterol concentration that resulted in less water penetration into the lamellar center.

Regarding the ternary mixtures, DPH lifetime values were correlated with packing order inferred based on steady-state anisotropy (Table 2). Thus, the lifetime of the PC-PS-Chol model indicated a higher packing order and implied reduced water molecule penetration into the lamellar depths. In these models, the effect of cholesterol on DPH lifetime was nonmonotonic and also exhibited a significant maximum near $33.3 \mathrm{~mol} \%$. A peak also appeared near $25 \mathrm{~mol} \%$, particularly in the PC-PS model.

To establish whether lamellar physiochemical properties affected the vesicle fusion process, the $\%$ leakage and $\%$ content mixing of the different models were determined (Fig. 3 and 4, respectively). Both parameters were determined as described by Talbot et al.20, and the results are summarized below.
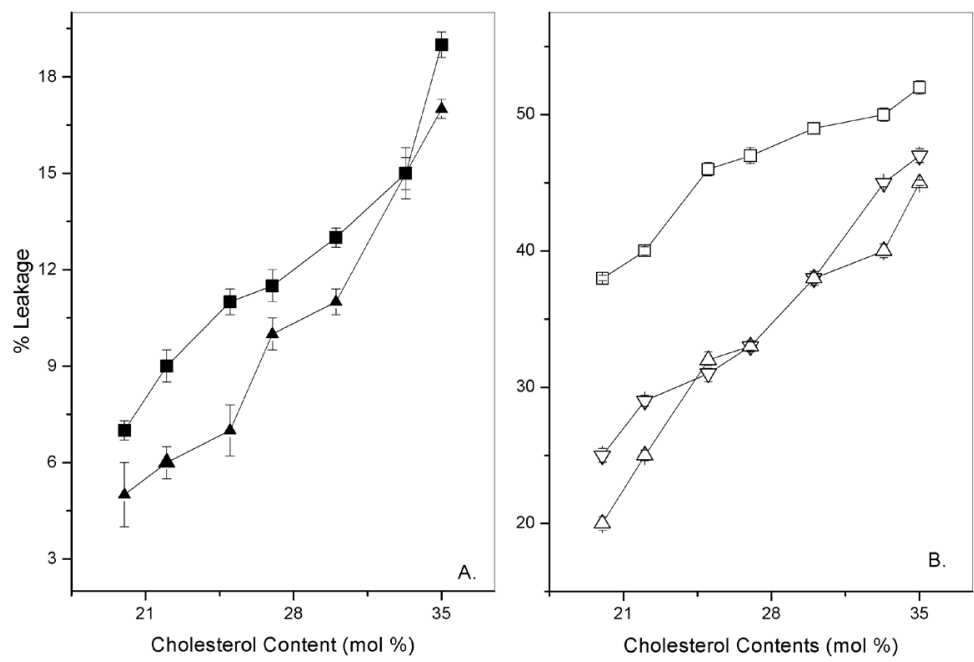

Figure 3: Effect of cholesterol on \% leakage. The vesicles contain - - DOPC, - $\mathbf{\Delta}$ - DSPC andB. - $\square$ $\mathrm{PC}: \mathrm{PE},-\Delta-\mathrm{PC}: \mathrm{SM}$, and $-\Delta-\mathrm{PC}: \mathrm{PS}$. Each point represents the average and standard error of triplicate data
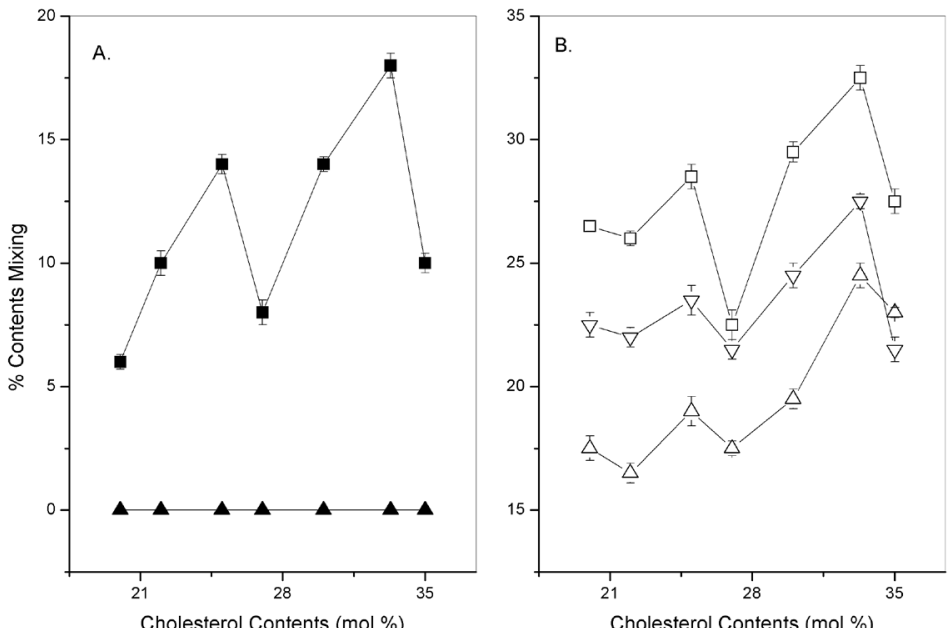

Figure 4: Effect of cholesterol on \% content mixing in vesicle-vesicle fusion. The vesicles contain - $\mathbf{m - D O P C}$, - $\mathbf{\Delta}$ DSPCand B - $\square$ - PC:PE, $-\Delta$ - PC:SM, and - $\Delta$-PC:PS. Each point represents the average and standard error of triplicate data. 


\section{$\%$ Leakage}

Similar leakage behavior was observed for the binary mixtures (Fig. $3 \mathrm{~A})$. In both mixtures containing DOPC-Chol and DSPC-Chol, the \% content mixing and $\%$ leakage increased with increasing cholesterol concentration. The leakage was proportional in both models, even after including that the DSPC mixture was below the phase transition temperature. Because no differences were observed between the two mixtures, the properties of the lamella were not significantly affected by this process; however, increases in cholesterol increased\% leakage.

In the ternary mixtures, an increase in leakage with increasing cholesterol content was also observed (Fig. 3B). However, for all cholesterol concentrations, the leakage was greater in the PC-PE-Chol compound model, then the PCSM-Chol and PC-PS-Chol mixtures. These phenomena indicate that in these models, the leakage process was also dependent on the physicochemical properties of the lamella and local variations caused by cholesterol. According to Haque et.al. (2001) $\frac{19}{}$, the high level of leakage observed is probably due to rupture under the osmotic stress imposed by PEG effect.

\section{$\%$ Mixture}

Fusion in the binary mixtures showed diametrically different behavior (Fig. 4). In the DSPC-Chol model, no mixing was observed at any cholesterol concentration. This behavior was also observed in the model that was under its phase transition temperature (the DPPC-Chol model, data not shown), and it is probable that the high degree of order in the gel phase state made fusion phenomena less likely. The presence of unsaturation in the DOPC-Chol mixtures increased the probability of fusion even at low percentages. Interestingly, the effect of cholesterol on the \% mixing content was nonmonotonic, and significant peaks were observed at 25 and $33.3 \mathrm{~mol} \%$ cholesterol. The presence of these peaks indicates that certain concentrations of cholesterol caused local effects that preferentially favored fusion phenomenon (Fig. 4A). At these concentrations super lattice theory predicts higher ordered/disordered domains proportions, then probably at these concentrations the fusion phenomenon is more likely.

In the ternary mixtures, the greatest percentage of fusion occurred in mixtures containing PC-PE-Chol, then the PC-SM-Chol and mixtures, and the lowest values were observed for the PC-PS-Chol mixtures. These findings are similar to those observed for leakage behavior and were more significantly different than those observed with the binary mixtures (Fig. 4B). In the DOPCChol mixture, increases in cholesterol increased the \% content mixing in a nonmonotonic manner and resulted in significant peaks from 25 to $33.3 \mathrm{~mol} \%$ cholesterol. In the three ternary models, cholesterol-mediated increases in the $\%$ mixing content were similar and approximately $5.5 \%$, lower than those observed in the DOPC-cholesterol model.

\section{DISCUSSION}

To study the effects of lamellar physicochemical properties on the fusion process, LUV models were selected to make negligible the curvature effect. To change the physicochemical properties of the membrane models, binary and ternary lipid mixtures were used. Moreover, different cholesterol concentrations were selected. In the binary model, lipid acyl chains of equal lengths with and without unsaturation (DOPC-Chol and DSPC-Chol, respectively) were selected. For the ternary models, PC-PE-Chol, PC-SM-Chol and PC-PS-Chol mixtures were examined.

Analysis of the binary models revealed that the vesicles containing saturated lipids (i.e., DSPC-Chol) exhibited a higher packing order in the lamellar hydrophobic core, less water content in the hydrophobic core, and slower hydration/water molecule dynamic into the hydrophilic-hydrophobic interphase than vesicles containing unsaturated lipids. Increased cholesterol content affected these properties. In general, increasing the cholesterol concentration increased water penetration, likely due to greater disorder. This pattern correlates with similar results obtained in a series of studies from our group $\underline{38}$. Regarding fusion, the $\%$ mixing and $\%$ leakage were studied. Fusion was observed only in the mixtures with DOPC-Chol, although leakage occurred in both mixtures (Fig.3A and 4A). Haque et al.(2001) 19 indicates that cholesterol addition has a dramatic influence on a many membrane models, destabilizing bilayers and making rupture more likely than fusion. This observation explains the leakage increase and the reduction in $\%$ mixing content. However, the nonmonotonic behavior of this fusion might be indicative of another phenomenon.

In the ternary mixtures, higher disorder in the center of the bilayer, increased hydration in this region and in the hydrophilic-hydrophobic interphase were observed. For these models, leakage was also greater than fusion, but these models exhibited significantly greater $\%$ mixing values than the binary models. Lentz et al.(2007) 15 indicated that PEG results in high leakage values in LUV models due to the more frequent rupture of vesicles than SUV models.

The \% content mixing also showed nonmonotonic behavior in these models. Local maxima were observed at 25 and $33.3 \mathrm{~mol} \%$ cholesterol. The latter peak was correlated with steady state and time-resolved fluorescence measurements.

We previously obtained similar results in other studies concerning these local maxima ${ }^{30}$, 31. This behavior can be explained in the context of the super lattice theory proposed by Somerharju et al. $\underline{26}, 27$. According to this theory, cholesterol is regularly distributed in hexagonal lattices that form regular domains that coexist with irregular domains. At concentrations at which local maxima are observed, greater proportions of ordered domains to disordered domains are present. These higher proportions of ordered domains result in a smaller amount of water in the lamella, and the water is redistributed to other areas, such as the interphases between the ordered and disordered domains. In contrast, small perturbations in lipid packing, "hydrophobic defects", and amphipathic "impurities" in different models ${ }^{39-42}$ have been reported to significantly lower the threshold for fusion.

In all studied models, increases in cholesterol generally favored the fusion process, and at specific critical concentrations, this behavior was enhanced, likely due to the formation of ordered/disordered interphases. These interphases might increase the probability of fusion because they are considered "defects" in the membrane.

However, models below their phase transition temperature, such as the DSPC-Chol model, displayed shorter lifetimes, although cholesterol resulted in a small local maximum at $33.3 \mathrm{~mol} \%$ (Table 1) and fusion did not occur. Therefore, "defects" or ordered/disordered domain interphases are not the only requirements for fusion; rather, the hydrophobic center of the lamella also requires essential features to stabilize the process. It is possible that the "defects" are only the starting point at which fusion begins.

To understand fusion phenomenon Churchward et al.(2008) ${ }^{43}$ suggested that first, a lipidic fusion site must exist in a liquid disordered state that is capable of rapidly forming high curvature structures as in regions of varied fluidity within single intact microdomains. Second, the fusion site must be organized as a discrete (liquid-disordered) lipid phase bounded by multiple liquid-ordered microdomains. Complementing this, Malinin et al. (2002) 16 concluded that the ability of closely juxtaposed bilayers to form an initial intermediate ("stalk") as soon as they come into close contact is not influenced by the osmotic stress of membrane curvature, although pore formation is critically dependent on such stress.

Upon analysis of the global results, we first noted that ternary mixtures exhibited greater $\%$ mixing content, particularly those containing PE. The effects of cholesterol on all ternary models mixtures were similar (resulting in increases of approximately $5.5 \%$ of mixing content). However, in the DOPC mixtures, although fusion was initially low, it significantly increased (up to approximately $11 \%$ ) with increasing cholesterol. Furthermore, models with higher fusion (the PC-PE-Chol model) exhibited lower hydration in both the body of the lamella and at the hydrophilic/hydrophobic interphase and also exhibited higher packing (longer life, higher GP and reduced anisotropy). Using the "stalk model" to explain fusion, we postulate that the formation of intermediate 1 (stalk) might be favored by the presence of "defects" at large proportions of the interphase, providing more ordered-disordered domains. However, to produce fusion, the stabilization of a new state (TMC intermediate) is required, and this state should have both a degree of membrane hydration and a level of lipid packing order that is sufficient to produce the fusion but not high enough to prevent it.

\section{ACKNOWLEDGMENT}

This work was supported by Fondo Nacional de Desarrollo Científico y Tecnológico, FONDECYT, Chile, grant N 11090443.

\section{REFERENCES}

1.- A. Grafmuller, J. Shillcock and R. Lipowsky, Biophysical journal, 96, 2658-2675 (2009).

2.- S. J. Marrink and A. E. Mark, Journal of the American Chemical Society, 125, 11144-11145 (2003)

3.- R. J. Lee and P. S. Low, The Journal of biological chemistry, 269, 31983204 (1994).

4.- S. L. Du, H. Pan, W. Y. Lu, J. Wang, J. Wu and J. Y. Wang, The Journal of pharmacology and experimental therapeutics, 322, 560-568 (2007). 
5.- W. H. De Jong and P. J. Borm, International journal of nanomedicine, $\mathbf{3}$, 133-149 (2008).

6.- H. Shibata, S. Nakagawa, T. Mayumi and Y. Tsutsumi, Biological \& pharmaceutical bulletin, 27, 1483-1488 (2004).

7.- G. Caracciolo, R. Caminiti, M. A. Digman, E. Gratton and S. Sanchez, The journal of physical chemistry. B, 113, 4995-4997 (2009).

8.- E. E. Oude Blenke, J. van den Dikkenberg, B. van Kolck, A. Kros and E. Mastrobattista, Nanoscale, 8, 8955-8965 (2016).

9.- K. Bacia, C. G. Schuette, N. Kahya, R. Jahn and P. Schwille, The Journal of biological chemistry, 279, 37951-37955 (2004).

10.- C. G. Schuette, K. Hatsuzawa, M. Margittai, A. Stein, D. Riedel, P. Kuster, M. Konig, C. Seidel and R. Jahn, Proc Natl Acad Sci USA, 101, 2858-2863 (2004).

11.- T. Lang, The Journal of physiology, 585, 693-698 (2007).

12.- M. Ma and D. Bong, Accounts of chemical research, 46, 2988-2997 (2013).

13.- B. Kolesinska, K. Eyer, T. Robinson, P. S. Dittrich, A. K. Beck, D. Seebach and P. Walde, Chemistry \& biodiversity, 12, 697-732 (2015).

14.- A. Herrmann, L. Pratsch, K. Arnold and G. Lassmann, Biochimica et biophysica acta, 733, 87-94 (1983).

15.- B. R. Lentz, European biophysics journal : EBJ, 36, 315-326 (2007).

16.- V. S. Malinin, P. Frederik and B. R. Lentz, Biophysical journal, 82, 20902100 (2002).

17.- V. S. Malinin and B. R. Lentz, Biophysical journal, 86, 2951-2964 (2004).

18.- V. S. Malinin and B. R. Lentz, Biochemistry, 41, 5913-5919 (2002).

19.- M. E. Haque, T. J. McIntosh and B. R. Lentz, Biochemistry, 40, 4340-4348 (2001).

20.- W. A. Talbot, L. X. Zheng and B. R. Lentz, Biochemistry, 36, 5827-5836 (1997).

21.- M. Tarshis, M. Salman and S. Rottem, Biophysical journal, 64, 709-715 (1993).

22.- J. Zhang, R. Xue, W. Y. Ong and P. Chen, Biophysical journal, 97, $1371-$ 1380 (2009).

23.- J. Chang, S. A. Kim, X. Lu, Z. Su, S. K. Kim and Y. K. Shin, Biophysical journal, 96, 1839-1846 (2009).

24.- B. Rituper, A. Flasker, A. Gucek, H. H. Chowdhury and R. Zorec, Cell calcium, 52, 250-258 (2012).

25.- M. R. Ali, K. H. Cheng and J. Huang, Proc Natl Acad Sci USA, 104, 5372 5377 (2007).

26.- P. J. Somerharju, J. A. Virtanen, K. K. Eklund, P. Vainio and P. K. Kinnunen, Biochemistry, 24, 2773-2781 (1985).

27.- J. A. Virtanen, M. Ruonala, M. Vauhkonen and P. Somerharju, Biochemistry, 34, 11568-11581 (1995).

28.- P. L. Chong, D. Tang and I. P. Sugar, Biophysical journal, 66, 2029-2038 (1994).

29.- D. Tang and P. L. Chong, Biophysical journal, 63, 903-910 (1992).

30.- F. J. Cuevas, D. M. Jameson and C. P. Sotomayor, Biochemistry, 45, 13855-13868 (2006).

31.- C. P. Sotomayor, L. F. Aguilar, F. J. Cuevas, M. K. Helms and D. M. Jameson, Biochemistry, 39, 10928-10935 (2000).

32.- R. D. Spencer; and G. Weber, Ann NY Acad Sci, 158, 361-376 (1969).

33.- E. Gratton, D. M. Jameson, G. Weber and B. Alpert, Biophysical journal, 45, 789-794 (1984)

34.- D. M. Jameson and T. L. Hazlett, in Biophysical and biochemical aspects of fluorescence spectroscopy, ed. T. G. Dewey, Plenum Press, New York, pp. 105-133 (1991).

35.- T. Parasassi, G. De Stasio, A. d'Ubaldo and E. Gratton, Biophysical journal, 57, 1179-1186 (1990).

36.- D. C. Mitchell and B. J. Litman, Biophysical journal, 75, 896-908 (1998)

37.- B. Cannon, A. Lewis, P. Somerharju, J. Virtanen, J. Huang and K. H. Cheng, The journal of physical chemistry. B, 114, 10105-10113 (2010).

38.- L. F. Aguilar, J. A. Pino, M. A. Soto-Arriaza, F. J. Cuevas, S. Sanchez and C. P. Sotomayor, PloS one, 7, e40254 (2012).

39.- D. E. Lee, M. G. Lew and D. J. Woodbury, Chemistry and physics of lipids, 166, 45-54 (2013).

40.- J. Lee and B. R. Lentz, Biochemistry, 36, 6251-6259 (1997).

41.- J. Lee and B. R. Lentz, Biochemistry, 36, 421-431 (1997).

42.- S. Martens, M. M. Kozlov and H. T. McMahon, Science, 316, 1205-1208 (2007).

43.- M. A. Churchward, T. Rogasevskaia, D. M. Brandman, H. Khosravani, P. Nava, J. K. Atkinson and J. R. Coorssen, Biophysical journal, 94, 39763986 (2008). 